\title{
SMALL EIGENVALUES OF LARGE HANKEL MATRICES: THE INDETERMINATE CASE
}

\author{
CHRISTIAN BERG, YANG CHEN and MOURAD E. H. ISMAIL*
}

\begin{abstract}
In this paper we characterize the indeterminate case by the eigenvalues of the Hankel matrices being bounded below by a strictly positive constant. An explicit lower bound is given in terms of the orthonormal polynomials and we find expressions for this lower bound in a number of indeterminate moment problems.
\end{abstract}

\section{Introduction}

Let $\alpha$ be a positive measure on $\mathrm{R}$ with infinite support and finite moments of all orders

$$
s_{n}=s_{n}(\alpha)=\int_{\mathrm{R}} x^{n} d \alpha(x) .
$$

With $\alpha$ we associate the infinite Hankel matrix $\mathscr{H}_{\infty}=\left\{H_{j k}\right\}$,

$$
H_{j k}=s_{j+k} .
$$

Let $\mathscr{H}_{N}$ be the $(N+1) \times(N+1)$ matrix whose entries are $H_{j k}, 0 \leq j, k \leq N$. Since $\mathscr{H}_{N}$ is positive definite, then all its eigenvalues are positive. The large $N$ asymptotics of the smallest eigenvalue, denoted as $\lambda_{N}$, of the Hankel matrix $\mathscr{H}_{N}$ has been studied in papers by Szegő [11], Widom and Wilf [13], Chen and Lawrence [6]. See also the monograph by Wilf [14]. All the cases considered by these authors are determinate moment problems, and it was shown in each case that $\lambda_{N} \rightarrow 0$, and asymptotic results were obtained about how fast $\lambda_{N}$ tends to zero.

The smallest eigenvalue can be obtained from the classical Rayleigh quotient:

$$
\lambda_{N}=\min \left\{\sum_{j=0}^{N} \sum_{k=0}^{N} s_{j+k} v_{j} v_{k}: \sum_{k=0}^{N} v_{j}^{2}=1, v_{j} \in \mathrm{R}, 0 \leq j \leq N\right\} .
$$

* This research is partially supported by the EPSRC GR/M16580 and NSF grant DMS 99-70865. Received August 1, 1999 
It follows that $\lambda_{N}$ is a decreasing function of $N$.

The main result of this paper is Theorem 1.1, which we state next.

THEOREM 1.1. The moment problem associated with the moments (1.1) is determinate if and only if $\lim _{N \rightarrow \infty} \lambda_{N}=0$.

We shall compare this result with a theorem of Hamburger [8, Satz XXXI], cf. [1, p. 83] or [10, p. 70].

Let $\mu_{N}$ be the minimum of the Hankel form $\mathscr{H}_{N}$ on the hyperplane $v_{0}=1$, i.e.

$$
\mu_{N}=\min \left\{\sum_{j=0}^{N} \sum_{k=0}^{N} s_{j+k} v_{j} v_{k}: v_{0}=1, v_{j} \in \mathrm{R}, 0 \leq j \leq N\right\},
$$

and let $\mu_{N}^{\prime}$ be the corresponding minimum for the moment sequence $s_{n}^{\prime}=$ $s_{n+2}, n \geq 0$, i.e.

$$
\begin{aligned}
\mu_{N}^{\prime} & =\min \left\{\sum_{j=0}^{N} \sum_{k=0}^{N} s_{j+k+2} v_{j}^{\prime} v_{k}^{\prime}: v_{0}^{\prime}=1, v_{j}^{\prime} \in \mathrm{R}, 0 \leq j \leq N\right\} \\
& =\min \left\{\sum_{j=0}^{N+1} \sum_{k=0}^{N+1} s_{j+k} v_{j} v_{k}: v_{0}=0, v_{1}=1, v_{j} \in \mathrm{R}, 0 \leq j \leq N+1\right\} .
\end{aligned}
$$

The theorem of Hamburger can be stated that the moment problem is determinate if and only if at least one of the $\operatorname{limits}_{\lim _{N \rightarrow \infty}} \mu_{N}, \lim _{N \rightarrow \infty} \mu_{N}^{\prime}$ are zero.

It is clear from (1.3), (1.4) that $\mu_{N} \geq \lambda_{N}$ and similarly $\mu_{N}^{\prime} \geq \lambda_{N+1}$. From these inequalities and Hamburger's theorem, we obtain the "only if" statement in Theorem 1.1. The "if" statement will be proved by finding a positive lower bound for the eigenvalues $\lambda_{N}$ in the indeterminate case, cf. Theorem 1.2 below.

We think that Theorem 1.1 has the advantage over the theorem of Hamburger that it involves only the moment sequence $\left(s_{n}\right)$ and not the shifted sequence $\left(s_{n+2}\right)$. In section 2 we give another proof of the "only if" statement to make the proof of Theorem 1.1 independent of Hamburger's theorem.

If

$$
\pi_{N}(x):=\sum_{j=0}^{N} v_{j} x^{j}, \quad v_{j} \in \mathrm{R}
$$

then a simple calculation shows that

$$
\sum_{0 \leq j, k \leq N} s_{j+k} v_{j} v_{k}=\int_{E} \pi_{N}^{2}(x) d \alpha(x),
$$


and

$$
\sum_{k=0}^{N} v_{k}^{2}=\int_{0}^{2 \pi}\left|\pi_{N}\left(\mathrm{e}^{i \theta}\right)\right|^{2} \frac{d \theta}{2 \pi}
$$

We could also study the reciprocal of $\lambda_{N}$ given by

$$
\frac{1}{\lambda_{N}}=\max \left\{\int_{0}^{2 \pi}\left|\pi_{N}\left(\mathrm{e}^{i \theta}\right)\right|^{2} \frac{d \theta}{2 \pi}: \pi_{N}, \int_{E} \pi_{N}^{2}(x) d \alpha(x)=1\right\} .
$$

Let $\left\{p_{k}\right\}$ denote the orthonormal polynomials with respect to $\alpha$, normalized so that $p_{k}$ has positive leading coefficient.

We recall that the moment problem is indeterminate, cf. [1], [10], if and only if there exists a non-real number $z_{0}$ such that

$$
\sum_{k=0}^{\infty}\left|p_{k}\left(z_{0}\right)\right|^{2}<\infty
$$

In the indeterminate case the series in (1.9) actually converges for all $z_{0}$ in C, uniformly on compact sets. In the determinate case the series in (1.9) diverges for all non-real $z_{0}$ and also for all real numbers except the at most countably many points, where $\alpha$ has a positive mass.

If we expand the polynomial (1.5) as a linear combination of the orthonormal system

$$
\pi_{N}(x)=\sum_{j=0}^{N} c_{j} p_{j}(x),
$$

then

$$
\begin{aligned}
\int_{0}^{2 \pi}\left|\pi_{N}\left(\mathrm{e}^{i \theta}\right)\right|^{2} \frac{d \theta}{2 \pi} & =\sum_{0 \leq j, k \leq N} c_{j} c_{k} \int_{0}^{2 \pi} p_{j}\left(\mathrm{e}^{i \theta}\right) p_{k}\left(\mathrm{e}^{-i \theta}\right) \frac{d \theta}{2 \pi} \\
& =\sum_{0 \leq j, k \leq N} \mathscr{K}_{j k} c_{j} c_{k},
\end{aligned}
$$

where we have defined

$$
\mathscr{K}_{j k}=\int_{0}^{2 \pi} p_{j}\left(\mathrm{e}^{i \theta}\right) p_{k}\left(\mathrm{e}^{-i \theta}\right) \frac{d \theta}{2 \pi} .
$$

Thus

$$
\frac{1}{\lambda_{N}}=\max \left\{\sum_{0 \leq j, k \leq N} \mathscr{K}_{j k} c_{j} c_{k}: c_{j}, \sum_{j=0}^{N} c_{j}^{2}=1\right\} .
$$


Since the eigenvalues of the matrix $\left(\mathscr{K}_{j k}\right)_{0 \leq j, k \leq N}$ are positive, and their sum is its trace, then

$$
\frac{1}{\lambda_{N}} \leq \sum_{k=0}^{N} \mathscr{K}_{k k}=\int_{0}^{2 \pi} \sum_{k=0}^{N}\left|p_{k}\left(\mathrm{e}^{i \theta}\right)\right|^{2} \frac{d \theta}{2 \pi} .
$$

Thus in the case of indeterminacy,

$$
\frac{1}{\lambda_{N}} \leq \int_{0}^{2 \pi} \sum_{k=0}^{\infty}\left|p_{k}\left(\mathrm{e}^{i \theta}\right)\right|^{2} \frac{d \theta}{2 \pi}<\infty,
$$

which shows that

$$
\lim _{N \rightarrow \infty} \lambda_{N} \geq\left(\int_{0}^{2 \pi} \frac{1}{\rho\left(\mathrm{e}^{i \theta}\right)} \frac{d \theta}{2 \pi}\right)^{-1},
$$

where

$$
\rho(z)=\left(\sum_{k=0}^{\infty}\left|p_{k}(z)\right|^{2}\right)^{-1} .
$$

We recall that for $z \in \mathrm{C} \backslash \mathbf{R}$ the number $\rho(z) /|z-\bar{z}|$ is the radius of the Weyl circle at $z$.

The above argument establishes the following result:

THEOREM 1.2. In the indeterminate case the smallest eigenvalue $\lambda_{N}$ of the Hankel matrix $\mathscr{H}_{N}$ is bounded below by the harmonic mean of the function $\rho$ along the unit circle.

We shall conclude this paper with examples, where we have calculated or estimated the quantity

$$
\rho_{0}=\int_{0}^{2 \pi} \sum_{k=0}^{\infty}\left|p_{k}\left(\mathrm{e}^{i \theta}\right)\right|^{2} \frac{d \theta}{2 \pi} .
$$

This will be done for the moment problems associated with the Stieltjes-Wigert polynomials, cf. [4], [12], the Al-Salam-Carlitz polynomials [2], the symmetrized version of polynomials of Berg-Valent ([3]) leading to a Freud-like weight [5], and the $q^{-1}$-Hermite polynomials of Ismail and Masson [9].

If we introduce the coefficients of the orthonormal polynomials as

$$
p_{k}(x)=\sum_{j=0}^{k} \beta_{k, j} x^{j}
$$


then

$$
\int_{0}^{2 \pi}\left|p_{k}\left(\mathrm{e}^{i \theta}\right)\right|^{2} \frac{d \theta}{2 \pi}=\sum_{j=0}^{k} \beta_{k, j}^{2}
$$

and therefore

$$
\rho_{0}=\sum_{k=0}^{\infty} \sum_{j=0}^{k} \beta_{k, j}^{2}
$$

Another possibility for calculating $\rho_{0}$ is to use the entire functions $B, D$ from the Nevanlinna matrix since it is well known that [1, p. 123]

$$
\sum_{k=0}^{\infty}\left|p_{k}(z)\right|^{2}=\frac{B(z) D(\bar{z})-D(z) B(\bar{z})}{z-\bar{z}} .
$$

It follows that

$$
\sum_{k=0}^{\infty}\left|p_{k}\left(\mathrm{e}^{i \theta}\right)\right|^{2}=\operatorname{Im}\left\{B\left(\mathrm{e}^{i \theta}\right) D\left(\mathrm{e}^{-i \theta}\right)\right\} / \sin \theta .
$$

\section{Indeterminate Moment Problems}

In this section we shall give a proof of Theorem 1.1 which is independent of Hamburger's result. We have already established that if $\lim _{N \rightarrow \infty} \lambda_{N}=0$, then the problem is determinate. We shall next prove that if $\lambda_{N} \geq \gamma$ for all $N$, where $\gamma>0$, then the problem is indeterminate. Since $1 / \lambda_{N} \leq 1 / \gamma$ for all $N$, and $1 / \lambda_{N}$ is the biggest eigenvalue of the positive definite matrix $\left(\mathscr{K}_{j k}\right)_{0 \leq j, k \leq N}$, we get

$$
\sum_{0 \leq j, k \leq N} \mathscr{K}_{j k} c_{j} \overline{c_{k}} \leq \frac{1}{\gamma} \sum_{j=0}^{N}\left|c_{j}\right|^{2}
$$

for all vectors $\left(c_{0}, \ldots, c_{N}\right) \in \mathrm{C}^{N+1}$. If we consider an arbitrary complex polynomial $p$ of degree $\leq N$ written as $p(x)=\sum_{k=0}^{N} c_{k} p_{k}(x)$, the inequality (2.1) can be formulated

$$
\int_{0}^{2 \pi}\left|p\left(\mathrm{e}^{i \theta}\right)\right|^{2} \frac{d \theta}{2 \pi} \leq \frac{1}{\gamma} \int|p(x)|^{2} d \alpha(x) .
$$


Let now $z_{0}$ be an arbitrary non-real number in the open unit disc. By the Cauchy integral formula

$$
p\left(z_{0}\right)=\frac{1}{2 \pi} \int_{0}^{2 \pi} \frac{p\left(\mathrm{e}^{i \theta}\right)}{\mathrm{e}^{i \theta}-z_{0}} \mathrm{e}^{i \theta} d \theta,
$$

and therefore

$$
\left|p\left(z_{0}\right)\right|^{2} \leq \int_{0}^{2 \pi}\left|p\left(\mathrm{e}^{i \theta}\right)\right|^{2} \frac{d \theta}{2 \pi} \int_{0}^{2 \pi} \frac{1}{\left|\mathrm{e}^{i \theta}-z_{0}\right|^{2}} \frac{d \theta}{2 \pi} .
$$

Combined with (2.2) we see that there is a constant $K$ such that for all complex polynomials $p$

$$
\left|p\left(z_{0}\right)\right|^{2} \leq K \int|p(x)|^{2} d \alpha(x),
$$

where $K=1 /\left(\gamma\left(1-\left|z_{0}\right|^{2}\right)\right)$.

This inequality implies indeterminacy in the following way. Applying it to the polynomial

$$
p(x)=\sum_{k=0}^{N} p_{k}\left(\overline{z_{0}}\right) p_{k}(x),
$$

we get

$$
\sum_{k=0}^{N}\left|p_{k}\left(z_{0}\right)\right|^{2} \leq K,
$$

and since $N$ is arbitrary, indeterminacy follows.

Remark. We see that the infinite positive definite matrix $\mathscr{K}_{\infty}=\left\{\mathscr{K}_{j, k}\right\}$ is bounded on $\ell^{2}$ if and only if $\lambda_{N} \geq \gamma$ for all $N$ for some $\gamma>0$. Furthermore $\mathscr{K}_{\infty}$ is of trace class if and only if $\rho_{0}<\infty$. The result of Theorem 1.1 can be reformulated to say that boundedness implies trace class for this family of operators.

\section{Examples}

We shall follow the notation and terminology for $q$-special functions as those in Gasper and Rahman [7].

Example 3.1 (The Stieltjes-Wigert Polynomials). These polynomials are orthonormal with respect to the weight function

$$
\omega(x)=\frac{k}{\sqrt{\pi}} \exp \left(-k^{2}(\log x)^{2}\right), \quad x>0,
$$


where $k>0$ is a positive parameter, cf. [4], [12]. They are given by

$$
p_{n}(x)=(-1)^{n} q^{\frac{n}{2}+\frac{1}{4}}(q ; q)_{n}^{-\frac{1}{2}} \sum_{k=0}^{n}\left(\begin{array}{l}
n \\
k
\end{array}\right)_{q} q^{k^{2}}\left(-q^{\frac{1}{2}} x\right)^{k}
$$

where we have defined $q=\exp \left\{-\left(2 k^{2}\right)^{-1}\right\}$.

It follows by (1.18) that

$$
\rho_{0}=\sum_{n=0}^{\infty} \frac{q^{n+\frac{1}{2}}}{(q ; q)_{n}} \sum_{k=0}^{n} q^{k(2 k+1)}\left(\begin{array}{l}
n \\
k
\end{array}\right)_{q}^{2}=\sum_{k=0}^{\infty} q^{2 k^{2}+k+\frac{1}{2}} \sum_{n=k}^{\infty} \frac{q^{n}}{(q ; q)_{n}}\left(\begin{array}{l}
n \\
k
\end{array}\right)_{q}^{2} .
$$

Putting $n=k+j$, the inner sum is

$$
\sum_{j=0}^{\infty} \frac{q^{k+j}}{(q ; q)_{k}^{2}} \frac{(q ; q)_{k+j}}{(q ; q)_{j}^{2}}=\frac{q^{k}}{(q ; q)_{k}} 2 \phi_{1}\left(q^{k+1}, 0 ; q ; q, q\right)
$$

and hence

$$
\rho_{0}=\sum_{k=0}^{\infty} \frac{q^{2\left(k+\frac{1}{2}\right)^{2}}}{(q ; q)_{k}}{ }_{2} \phi_{1}\left(0, q^{k+1} ; q ; q, q\right) .
$$

We can obtain another expression for $\rho_{0}$. We apply the transformation $[7$, (III.5)]

$$
{ }_{2} \phi_{1}(a, b ; c ; q, z)=\frac{(a b z / c ; q)_{\infty}}{(b z / c ; q)_{\infty}} \phi_{2}(a, c / b, 0 ; c, c q / b z ; q, q)
$$

to see that

$$
\sum_{n=k}^{\infty} \frac{q^{n}}{(q ; q)_{n}}\left(\begin{array}{l}
n \\
k
\end{array}\right)_{q}^{2}=\frac{1}{(q ; q)_{\infty}} \sum_{j=0}^{k} \frac{q^{k+j}}{(q ; q)_{j}^{2}}
$$

We then find

$$
\rho_{0}=\frac{1}{(q ; q)_{\infty}} \sum_{k=0}^{\infty} q^{2\left(k+\frac{1}{2}\right)^{2}} \sum_{j=0}^{k} \frac{q^{j}}{(q ; q)_{j}^{2}} .
$$

A formula more general than (3.6) is

$$
\sum_{n=k}^{\infty} \frac{\omega^{n}}{(q ; q)_{n}}\left(\begin{array}{l}
n \\
k
\end{array}\right)_{q}^{2}=\frac{1}{(\omega ; q)_{\infty}} \sum_{j=0}^{k} \frac{(\omega ; q)_{j} \omega^{2 k-j}}{(q ; q)_{j}(q ; q)_{k-j}^{2}}
$$


and is stated in [2]. This more general identity also follows from (3.5) and the simple observation

$$
\frac{\left(q^{-k} ; q\right)_{j}}{\left(q^{1-k} / \omega ; q\right)_{j}}=\frac{(q ; q)_{k}(\omega ; q)_{k-j}}{(\omega ; q)_{k}(q ; q)_{k-j}}(\omega / q)^{j}
$$

We have numerically computed the smallest eigenvalue of the Hankel matrix of various dimensions with the Stieltjes-Wigert weight from which we extrapolate to determine the smallest eigenvalue $s=\lim _{N \rightarrow \infty} \lambda_{N}$ of the infinite Hankel matrix for different values of $q$. This is then compared with the numerically computed lower bound $l=1 / \rho_{0}$. For $q=\frac{1}{2}$ we have $s=0.3605 \ldots, l=$ $0.3435 \ldots$ The percentage error $100(s-l) / s$ is plotted for various values of $q$ and is shown in figure 1 .

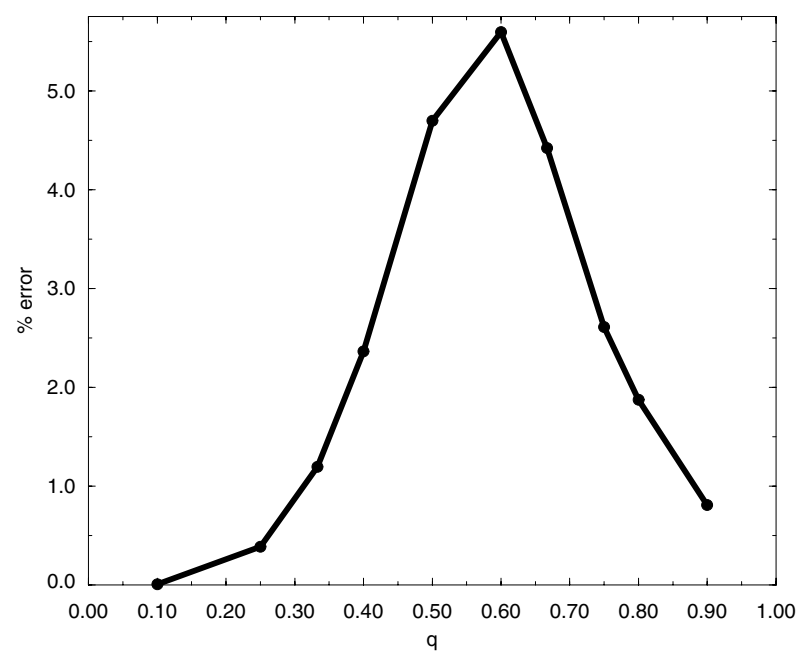

FIGURE 1. Percentage error plotted for various values of $q$.

EXAMPLE 3.2 (Al-Salam-Carlitz polynomials). The Al-Salam-Carlitz polynomials were introduced in [2]. We consider the indeterminate polynomials $V_{n}^{(a)}(x ; q)$, where $0<q<1$ and $q<a<1 / q$, cf. [3]. For the corresponding orthonormal polynomials $\left\{p_{k}\right\}$ we have by $[3,(4.24)]$

$$
\begin{aligned}
& \sum_{k=0}^{\infty}\left|p_{k}\left(\mathrm{e}^{i \theta}\right)\right|^{2} \\
& \quad=\frac{\left(q \mathrm{e}^{i \theta}, q \mathrm{e}^{-i \theta} ; q\right)_{\infty}}{(a q, q, q ; q)_{\infty}}{ }_{3} \phi_{2}\left(\mathrm{e}^{i \theta}, \mathrm{e}^{-i \theta}, a q ; q \mathrm{e}^{i \theta}, q \mathrm{e}^{-i \theta} ; q, q / a\right) .
\end{aligned}
$$


Therefore

$$
\rho_{0}=\int_{0}^{2 \pi} \sum_{k=0}^{\infty}\left|p_{k}\left(\mathrm{e}^{i \theta}\right)\right|^{2} \frac{d \theta}{2 \pi}=\frac{1}{(a q, q, q ; q)_{\infty}} \sum_{n=0}^{\infty} I_{n} \frac{(a q ; q)_{n}}{(q ; q)_{n}}\left(\frac{q}{a}\right)^{n},
$$

where

$$
\begin{aligned}
I_{n} & =\int_{0}^{2 \pi} \frac{\left(\mathrm{e}^{i \theta}, \mathrm{e}^{-i \theta} ; q\right)_{\infty}}{\left(1-q^{n} \mathrm{e}^{i \theta}\right)\left(1-q^{n} \mathrm{e}^{-i \theta}\right)} \frac{d \theta}{2 \pi} \\
& =\int_{|z|=1} \frac{(z, 1 / z ; q)_{\infty}}{\left(1-q^{n} z\right)\left(1-q^{n} / z\right)} \frac{d z}{2 \pi i z} .
\end{aligned}
$$

Recall the Jacobi triple product identity [7],

$$
j(z):=(q, z, 1 / z ; q)_{\infty}=\sum_{k=-\infty}^{\infty} c_{k} z^{k}
$$

with

$$
c_{k}=(-1)^{k}\left[q^{k(k+1) / 2}+q^{k(k-1) / 2}\right] .
$$

Note that $c_{k}=c_{-k}$.

Using the partial fraction decomposition

$$
\frac{q^{n}}{1-q^{n} z}-\frac{q^{-n}}{1-q^{-n} z}=\frac{1-q^{2 n}}{\left(1-q^{n} z\right)\left(z-q^{n}\right)}
$$

we find by the residue theorem and the Jacobi triple product identity (3.11) that for $n \geq 1, I_{n}$ is given by

$$
\begin{aligned}
\left(1-q^{2 n}\right) & (q ; q)_{\infty} I_{n} \\
& =q^{n} \operatorname{Res}\left(\frac{j(z)}{1-q^{n} z}, z=0\right)-q^{-n} \operatorname{Res}\left(\frac{j(z)}{1-q^{-n} z}, z=0\right) \\
& =q^{n} \sum_{k=0}^{\infty} q^{n k} c_{-k-1}-q^{-n} \sum_{k=0}^{\infty} q^{-n k} c_{-k-1} \\
& =\sum_{k=1}^{\infty}\left(q^{n k}-q^{-n k}\right) c_{k},
\end{aligned}
$$


while for $n=0, I_{0}$ is

$$
\begin{aligned}
(q ; q)_{\infty} I_{0} & =\int_{|z|=1} \frac{j(z)}{(1-z)(z-1)} \frac{d z}{2 \pi i}=-\operatorname{Res}\left(\frac{j(z)}{(1-z)^{2}}, z=0\right) \\
& =-\sum_{k=0}^{\infty}(k+1) c_{-k-1}=\sum_{k=0}^{\infty}(-1)^{k} q^{k(k+1) / 2} .
\end{aligned}
$$

The conclusion is

$$
\begin{aligned}
& I_{0}=\frac{1}{(q ; q)_{\infty}} \sum_{k=0}^{\infty}(-1)^{k} q^{k(k+1) / 2}, \\
& I_{n}=\frac{1}{\left(1-q^{2 n}\right)(q ; q)_{\infty}} \sum_{k=1}^{\infty} c_{k}\left(q^{n k}-q^{-n k}\right), \quad n \geq 1
\end{aligned}
$$

The above formulas can be further simplified. Using the Jacobi triple product identity (3.11) we find for integer values of $n$

$$
\sum_{k=-\infty}^{\infty}(-1)^{k} q^{n k} q^{\left(\begin{array}{l}
k \\
2
\end{array}\right)}=0
$$

hence

$$
\sum_{k=0}^{\infty}(-1)^{k} q^{n k} q^{\left(\begin{array}{l}
k \\
2
\end{array}\right)}=-\sum_{k=1}^{\infty}(-1)^{k} q^{-n k} q^{\left(\begin{array}{c}
k+1 \\
2
\end{array}\right)}, \quad n=0, \pm 1, \ldots
$$

This analysis implies

$$
(q ; q)_{\infty}\left(1-q^{2 n}\right) I_{n}=2 \sum_{k=1}^{\infty}(-1)^{k} q^{\left(\begin{array}{c}
k \\
2
\end{array}\right)}\left[q^{n k}-q^{-n k}\right] .
$$

Thus we have established the representation for $n \geq 1$

$$
I_{n}=\frac{2 q^{-n}}{(q ; q)_{\infty}} \sum_{k=1}^{\infty}(-1)^{k-1} q^{\left(\begin{array}{l}
k \\
2
\end{array}\right)} \frac{\sin (n k \tau)}{\sin (n \tau)}, \quad q=e^{-i \tau} .
$$

It is clear that $I_{0}$ is the limiting case of $I_{n}$ as $n \rightarrow 0$. The representation (3.16) indicates that $I_{n}$ is a theta function evaluated at the special point $n \tau$, hence we do not expect to find a closed form expression for $I_{n}$.

Example 3.3 (Freud-like weight). In [3] Berg-Valent found the Nevanlinna matrix in the case of the indeterminate moment problem corresponding 
to a birth and death process with quartic rates. Later Chen and Ismail, cf. [5], considered the corresponding symmetrized moment problem, found the Nevanlinna matrix and observed that there are solutions which behave as the Freud weight $\exp (-\sqrt{|x|})$. In particular they found the entire functions

$$
B(z)=-\delta_{0}\left(K_{0} \sqrt{z / 2}\right), \quad D(z)=\frac{4}{\pi} \delta_{2}\left(K_{0} \sqrt{z / 2}\right),
$$

where

$$
\begin{aligned}
\delta_{l}(z) & =\sum_{n=0}^{\infty} \frac{(-1)^{n}}{(4 n+l) !} z^{4 n+l}, \quad l=0,1,2,3, \\
K_{0} & =\frac{\Gamma(1 / 4) \Gamma(5 / 4)}{\sqrt{\pi}} .
\end{aligned}
$$

Note that

$$
\begin{aligned}
& \delta_{0}(z)=\frac{1}{2}[\cosh (z \sqrt{i})+\cos (z \sqrt{i})], \\
& \delta_{2}(z)=\frac{1}{2 i}[\cosh (z \sqrt{i})-\cos (z \sqrt{i})] .
\end{aligned}
$$

If $\omega:=\exp (i \pi / 4)=(1+i) / \sqrt{2}$, then a simple calculation shows that

$$
\begin{aligned}
B(x) D(y)-D(x) B(y)=\frac{-2 i}{\pi} & {\left[\cos \left(\omega^{3} K_{0} \sqrt{x / 2}\right) \cos \left(\omega K_{0} \sqrt{y / 2}\right)\right.} \\
& \left.-\cos \left(\omega^{3} K_{0} \sqrt{y / 2}\right) \cos \left(\omega K_{0} \sqrt{x / 2}\right)\right] .
\end{aligned}
$$

If $x=\mathrm{e}^{i \theta}$, and $y=\mathrm{e}^{-i \theta}$, then we linearize the products of cosines and find that the right-hand side of (3.22) is

$$
\begin{aligned}
& \frac{-i}{\pi}\left\{\cos \left[K_{0}\left(\omega^{3} e^{i \theta / 2}+\omega e^{-i \theta / 2}\right) / \sqrt{2}\right]+\cos \left[K_{0}\left(\omega^{3} e^{i \theta / 2}-\omega e^{-i \theta / 2}\right) / \sqrt{2}\right]\right. \\
& \left.-\cos \left[K_{0}\left(\omega^{3} e^{-i \theta / 2}+\omega e^{i \theta / 2}\right) / \sqrt{2}\right]-\cos \left[K_{0}\left(\omega^{3} e^{-i \theta / 2}-\omega e^{i \theta / 2}\right) / \sqrt{2}\right]\right\} .
\end{aligned}
$$

We now combine the first and third terms, then combine the second and fourth terms and apply the addition theorem for trigonometric functions. We then see that the above is

$$
\begin{aligned}
& \frac{2 i}{\pi}\left\{\sinh \left[K_{0} \cos (\theta / 2)\right] \sinh \left[K_{0} \sin (\theta / 2)\right]\right. \\
& \left.+\sin \left[K_{0} \cos (\theta / 2)\right] \sin \left[K_{0} \sin (\theta / 2)\right]\right\} .
\end{aligned}
$$


Thus we have proved that

$$
\begin{aligned}
& \frac{B\left(\mathrm{e}^{i \theta}\right) D\left(\mathrm{e}^{-i \theta}\right)-B\left(\mathrm{e}^{-i \theta}\right) D\left(\mathrm{e}^{i \theta}\right)}{\mathrm{e}^{i \theta}-\mathrm{e}^{-i \theta}} \\
& =\frac{1}{\pi \sin \theta}\left\{\sinh \left[K_{0} \cos (\theta / 2)\right] \sinh \left[K_{0} \sin (\theta / 2)\right]\right. \\
& \left.\quad+\sin \left[K_{0} \cos (\theta / 2)\right] \sin \left[K_{0} \sin (\theta / 2)\right]\right\} .
\end{aligned}
$$

Thus in the case under consideration, after some straightforward calculations and the evaluation of a beta integral, we obtain

$$
\begin{aligned}
\rho_{0} & =\int_{0}^{2 \pi} \sum_{n=0}^{\infty}\left|p_{n}\left(\mathrm{e}^{i \theta}\right)\right|^{2} \frac{d \theta}{2 \pi} \\
& =\frac{K_{0}^{2}}{\pi} \sum_{m, n \geq 0, m+n \text { even }} \frac{\left(K_{0} / 2\right)^{2 m+2 n}}{(2 m+1)(2 n+1) m ! n !(m+n) !} .
\end{aligned}
$$

EXAmPLe 3.4 ( $q^{-1}$-Hermite polynomials). Ismail and Masson [9] proved that for this moment problem the functions $B$ and $D$ are given by

$$
\begin{aligned}
B(\sinh \xi) & =-\frac{\left(q e^{2 \xi}, q e^{-2 \xi} ; q^{2}\right)_{\infty}}{\left(q, q ; q^{2}\right)_{\infty}}, \\
D(\sinh \xi) & =\frac{\sinh \xi}{(q ; q)_{\infty}}\left(q^{2} e^{2 \xi}, q^{2} e^{-2 \xi} ; q^{2}\right)_{\infty},
\end{aligned}
$$

$[9,(5.32)],[9,(5.36)]$; respectively. Ismail and Masson also showed that $[9$, $(6.25)]$

$$
\begin{aligned}
& B(\sinh \xi) D(\sinh \eta)-B(\sinh \eta) D(\sinh \xi) \\
& =\frac{-e^{\eta}}{2(q ; q)_{\infty}} \prod_{n=0}^{\infty}\left[1-2 e^{-\eta} q^{n} \sinh \xi-e^{-2 \eta} q^{2 n}\right] \\
& \cdot\left[1+2 e^{\eta} q^{n+1} \sinh \xi-e^{2 \eta} q^{2 n+2}\right] .
\end{aligned}
$$

We rewrite the infinite product as

$$
\prod_{n=0}^{\infty} a_{n} b_{n}=a_{0} \prod_{n=1}^{\infty} a_{n} b_{n-1},
$$


and with $\sinh \xi=e^{i \theta}$ and $\sinh \eta=e^{-i \theta}$ we get the following representation (3.28)

$$
\begin{aligned}
& \frac{B\left(e^{i \theta}\right) D\left(e^{-i \theta}\right)-B\left(e^{-i \theta}\right) D\left(e^{i \theta}\right)}{e^{i \theta}-e^{-i \theta}} \\
& =\frac{1}{(q ; q)_{\infty}} \prod_{n=1}^{\infty}\left[1+4 q^{n}-2 q^{2 n}+4 q^{3 n}+q^{4 n}-8 q^{2 n} \cos (2 \theta)\right] \\
& =\frac{1}{(q ; q)_{\infty}} \prod_{n=1}^{\infty}\left[\left(1+q^{n}\right)^{4}-16 q^{2 n} \cos ^{2} \theta\right] .
\end{aligned}
$$

Writing the infinite product as a power series in $\cos ^{2} \theta$ and using

$$
\int_{-\pi}^{\pi} \cos ^{2 k} \theta \frac{d \theta}{2 \pi}=2^{-2 k}\left(\begin{array}{c}
2 k \\
k
\end{array}\right)
$$

we evaluate the integral of (3.28) with respect to $d \theta / 2 \pi$ as

$$
\rho_{0}=\frac{(-q ; q)_{\infty}^{4}}{(q ; q)_{\infty}} \sum_{k=0}^{\infty}\left(\begin{array}{c}
2 k \\
k
\end{array}\right) \sum_{1 \leq n_{1}<\cdots<n_{k}} \frac{(-2)^{2 k} q^{2\left(n_{1}+\cdots+n_{k}\right)}}{\left[\left(1+q^{n_{1}}\right) \ldots\left(1+q^{n_{k}}\right)\right]^{4}} .
$$

The formula (3.28) can be transformed further by putting $\cos ^{2} \psi=-\cos \theta$ and $p^{2}=q$, because then

$$
\prod_{n=1}^{\infty}\left[\left(1+q^{n}\right)^{2}+4 q^{n} \cos \theta\right]=\prod_{n=1}^{\infty}\left[1+p^{4 n}-2 p^{2 n} \cos (2 \psi)\right]
$$

can be expressed by means of the theta function $\vartheta_{1}(p ; \psi)$. We find

$$
\prod_{n=1}^{\infty}\left[\left(1+q^{n}\right)^{2}+4 q^{n} \cos \theta\right]=\frac{1}{(q ; q)_{\infty}} \sum_{n=0}^{\infty}(-1)^{n} q^{\left(\begin{array}{c}
n+1 \\
2
\end{array}\right)} U_{2 n}(\cos \psi)
$$

where

$$
U_{2 n}(\cos \psi)=\frac{\sin (2 n+1) \psi}{\sin \psi}
$$

is the Chebyshev polynomium of the second kind given by

$$
U_{2 n}(x)=\sum_{k=0}^{n}\left(\begin{array}{c}
2 n+1 \\
2 k+1
\end{array}\right)(-1)^{k} x^{2(n-k)}\left(1-x^{2}\right)^{k}
$$

Similarly putting $\cos ^{2} \varphi=\cos \theta$ we find

$$
\prod_{n=1}^{\infty}\left[\left(1+q^{n}\right)^{2}-4 q^{n} \cos \theta\right]=\frac{1}{(q ; q)_{\infty}} \sum_{n=0}^{\infty}(-1)^{n} q^{\left(\begin{array}{c}
n+1 \\
2
\end{array}\right)} U_{2 n}(\cos \varphi)
$$


If we let $U_{n}^{*}$ be the polynomial of degree $n$ such that $U_{2 n}(x)=U_{n}^{*}\left(x^{2}\right)$, we get

$$
\begin{aligned}
& \frac{B\left(e^{i \theta}\right) D\left(e^{-i \theta}\right)-B\left(e^{-i \theta}\right) D\left(e^{i \theta}\right)}{e^{i \theta}-e^{-i \theta}} \\
& =\frac{1}{(q ; q)_{\infty}^{2}} \sum_{n, m=0}^{\infty}(-1)^{m} q^{\left(\begin{array}{c}
n+1 \\
2
\end{array}\right)+\left(\begin{array}{c}
m+1 \\
2
\end{array}\right)} U_{n}^{*}(-\cos \theta) U_{m}^{*}(\cos \theta)
\end{aligned}
$$

For non-negative integers $k, l, r$ we have

$$
\begin{aligned}
C(k, l, r): & =\frac{1}{2 \pi} \int_{0}^{2 \pi}(1+\cos \theta)^{k}(1-\cos \theta)^{l} \cos ^{r} \theta d \theta \\
& =\frac{2^{k+l}}{\pi}(-1)^{r} B\left(k+\frac{1}{2}, l+\frac{1}{2}\right){ }_{2} F_{1}\left(k+\frac{1}{2},-r ; k+l+1 ; 2\right),
\end{aligned}
$$

which gives

$$
\begin{aligned}
& \frac{1}{2 \pi} \int_{0}^{2 \pi} U_{n}^{*}(-\cos \theta) U_{m}^{*}(\cos \theta) d \theta \\
& \quad=\sum_{k=0}^{n} \sum_{l=0}^{m}\left(\begin{array}{c}
2 n+1 \\
2 k+1
\end{array}\right)\left(\begin{array}{c}
2 m+1 \\
2 l+1
\end{array}\right)(-1)^{n+l} C(k, l, n+m-k-l) .
\end{aligned}
$$

Putting these formulas together we get a 5-fold sum for $\rho_{0}$.

AcKnowledgement. The authors would like to thank Mr. N. D. Lawrence for supplying the numerical data and the graph.

\section{REFERENCES}

1. Akhiezer, N. I., The Classical Moment Problem and Some Related Questions in Analysis, English translation, Oliver and Boyd, Edinburgh, 1965.

2. Al-Salam, W. A. and Carlitz, L., Some orthogonal q-polynomials, Math. Nachr. 30 (1965), 47-61.

3. Berg, C., and Valent, G., The Nevanlinna parametrization for some indeterminate Stieltjes moment problems associated with birth and death processes, Methods and Applications of Analysis 1 (1994), 169-209.

4. Chihara, T. S., An Introduction to Orthogonal Polynomials, Gordon and Breach, New York, 1978.

5. Chen, Y. and Ismail, M. E. H., Some indeterminate moment problems and Freud-like weights, Constr. Approx. 14 (1998), 439-458.

6. Chen, Y. and Lawrence, N. D., Small eigenvalues of large Hankel matrices, J. Phys. A 32 (1999), 7305-7315.

7. Gasper, G. and Rahman, M., Basic Hypergeometric Series, Cambridge University Press, Cambridge, 1990. 
8. Hamburger, H., Über eine Erweiterung des Stieltjesschen Momentenproblems, Math. Ann. 81 (1920), 235-319, 82, 120-164, 168-187.

9. Ismail, M. E. H. and Masson, D. R., q-Hermite polynomials, biorthogonal rational functions, and q-beta integrals, Trans. Amer. Math. Soc. 346 (1994), 63-116.

10. Shohat, J. and Tamarkin, J. D., The Problem of Moments, Revised edition, American Mathematical Society, Providence, 1950.

11. Szegő, G., On some Hermitian forms associated with two given curves of the complex plane, Collected papers (volume 2), 666-678. Birkhaüser, Boston, Basel, Stuttgart, 1982.

12. Szegő, G., Orthogonal Polynomials, Fourth Edition, American Mathematical Society, Providence, 1975.

13. Widom, H. and Wilf, H. S., Small eigenvalues of large Hankel matrices, Proc. Amer. Math. Soc. 17 (1966), 338-344.

14. Wilf, H. S., Finite Sections of some Classical Inequalities, Springer, Berlin, Heidelberg, New York, 1970.

DEPARTMENT OF MATHEMATICS

UNIVERSITY OF COPENHAGEN

UNIVERSITETSPARKEN 5

DK-2100 COPENHAGEN

DENMARK

E-mail: berg@math.ku.dk

DEPARTMENT OF MATHEMATICS

UNIVERSITY OF SOUTH FLORIDA

TAMPA, FLORIDA 33620-5700

USA

E-mail: ismail@math.usf.edu
DEPARTMENT OF MATHEMATICS

IMPERIAL COLLEGE

180 QUEEN'S GATE

SW7 2BZ LONDON

ENGLAND

E-mail: y.chen@ic.ac.uk 